\title{
Inhalt, Vol. 17, No. 6, 1994
}

\section{Contents}

Übersichtsarbeit

Modulation der «Multidrug-Resistenz» von Tumoren durch Dexverapamil

Keilhauer, G., Romerdahl, C, Kupper, H., Schlick, E 572

Review Article

Modulation of Multidrug-Resistant Tumors by Dexverapamil

Keilhauer, G, Romerdahl, C, Kupper, H., Schlick, E 572

Originalarbeiten

Lokalisiertes Prostatakarzinom: Der Nutzen der serien-mäßigen Bestimmung des prostataspezifíschen Antigens während der Strahlentherapie - Ein Update

Vijayakumar, S., Karrison, T., Quadri, S. R, Chan S. K., Haraf, D., Pandya, K., Houghton, A., Rubin, S., Kalokhe, U., Halpern, H., Muller-Runkel, R., Sutton, H., Awan A., Weichselbaum, R 578

Prostatakarzinom Stadium Dl - gleichwertige Ergebnisse mit Strahlen- und Hormontherapie versus radikale Prostatektomie, Strahlen- und Hormontherapie (?)

Wiegel, T., Bressel, M., Schmidt, R 586

Chirurgische Therapie und Prognosefaktoren beim medullaren Schilddrüsenkarzinom. Aktuelle

Erhebung der Deutschen Studiengruppe «Medulläres Schilddrüsenkarzinom»

Winter, X, Raue, R, und die German MTC Study Group 594

In $\mathrm{mi}$ I in : $1 \mathrm{l} \mathrm{i} \mathrm{Ik}$ he Wachstumsfaktoren (IGF)-I und -II und IGF-Bindeprotein in der

Prolaktinomzyste

Zumkeller, W., Sääf, M., Rähn, T. 600

Erfahrungen mit einem neuen Пexiblen Applikatorsystem zur intrazervikalen Strahlentherapie des Zervixkarzinoms

Meden, H., Busch, M., Bartels, VS., Tsikuras, P., Rath, W.,

Dühmke, E., Kuhn, W. 604

Original Articles

Localized Prostate Cancer: Use of Serial Prostate-Specific Antigen Measurements during

Radiation Therapy - An Update

Vijayakumar, S., Karrison, T., Quadri, S. R, Chan S. K., Haraf, D.,

Pandya, K., Houghton, A., Rubin, S., Kalokhe, U, Halpern, H,

Muller-Runkel, R., Sutton, H., Awan A., Weichselbaum, R 578

Stage Dl Prostatic Cancer - Equivalent Results with Radiotherapy and Hormonal Therapy versus

Radical Prostatectomy, Radiotherapy and Hormonal Therapy (?)

Wiegel, T., Bressel, M., Schmidt, R 586

Surgical Therapy and Prognostic Factors in Medullary Thyroid Carcinoma (MTC). Current

Evaluation of the German MTC Study Group 
Winter, J., Raue, E, and the German MTC Study Group 594

Insulin-Like Growth Factors (IGF)-I and -II and IGF-Binding Proteins in the Prolactinoma Cyst Fluid

Zumkeller, W, Sääf, M., Rähn, T. 600

Experience with a New Flexible Applicator System for Intracavitary Radiotherapy of Cervical

Cancer

Meden, H, Busch, M., Bartels, U, Tsikuras, P., Rath, W,

Dühmke, E., Kuhn, W. 604

Briefe an die Herausgeber

Hiddemann, W., zu:

Fenchel K, et al: Fludarabin ist eine effektive Substanz in

der Therapie des Immunozytoms 608

Hoffmann, W., Kortmann, R.D., Bamberg, M., zu:

Schroder M, Queißer W: Behandlung von

Desmoidtumoren 609

Letters to the Editors

Hiddemann, W, concerning:

Fenchel K, et al: Fludarabine is an effective agent in

immunocytic lymphoma 608

Hoffmann, W, Kortmann, R. D., Bamberg, M., concerning:

Schroder M, Queißer W: Management of

desmoid tumours 609

Fortsetzung aufSeite 570

Continued on page 570

Band 17, Heft 6, Dezember 1994

International Journal for Cancer Research and Treatment

Inhalt

Contents

Kommentar

Commentary

Verstärkung toxischer Wïrkungen durch 5-Fluorouracil und Intensification of Toxic Effects by 5Fluorouracil and Salts

Salze der Folinsäure of Foiinic Acid

Hartenstein, R 610 Hartenstein, R 610

Klinische Information

Clinical Information

Nutzen und Risiken der kombinierten Radiochemotherapie Advantages and Risks of Combined

Radiochemotherapy

Hinkelbein, W. 612 Hinkelbein, W. 612

Inhalt und Perspektiven der Krebsforschung

zur Hausen, $\mathrm{H}$

Subject and Prospects of Cancer Research 
618 zur Hausen, $\mathrm{H}$

$61<\mathrm{S}$

\section{Meinung}

Alternative Strategien in der medikamentösen Tumortherapie: sozialanthropologische Dimension Kappauf, H., Kaiser, G., Gallmeier, W.M

624

Opinion

Alternative Strategies in Medical Oncology: Social-Anthropological Dimension

Kappauf, H., Kaiser, G., Gallmeier, W. M

624

Implementierung der Good Clinical Practice (GCP)-Empfehlungen in Deutschland Queißer,W.

Implementation of the Good Clinical Practice (GCP) Recommendations in Germany 630 Queißer, W.

630

Kongreßberichte 636 Congress Reports

Buchbesprechung 640 Book Review ...

636

640

Industrieforum 641 Industrial Forum

641

Mitteilungen onkologischer Gesellschaften 643 Reports of Oncological Societies 643

Impressum $567 \quad$ Imprint $\quad 567$

Autorenverzeichnis $1994 \quad 645 \quad$ Author Index 1994645

Sachwortregister $1994 \quad 647 \quad$ Subject Index $1994 \quad 649$

Jahres-Inhalt 1994 Ill (nach 650) Complete Contents $1994 \quad$ VII (following 650)

Hinweise für Autoren 651 Instructions to Authors 652

Bibliographischer Hinweis: Inhaltsverzeichnisse dieser Zeitschrift erscheinen regelmäßig in current contents ${ }^{\circledR}$ sowie in anderen bibliographischen Diensten. 